\title{
AN UNUSUAL CASE OF THE GRASP REFLEX, WITH SOME OBSERVATIONS ON THE VOLITIONAL AND REFLEX COMPONENTS
}

\author{
BY
}

\section{A. M. STEWART-WALLACE}

(ReCeIVed 12TH December, 1938)

IN 1927 Adie and Critchley, in their review of the grasp reflex, concluded that the closure of the hand following a stroke across the palm is an involuntary reflex response to a tactile stimulus. The whole hand, arm, or even the body may sometimes turn reflexly in the direction of the stimulus. "Forced groping and grasping" after objects merely coming into the neighbourhood of the hand they also believed is involuntary and an automatic reaction under control of a mechanism on a high physiological level, deprived of the normal control of a still higher and less primitive centre.

Walshe and Robertson in 1933 suggested the phenomena included in the term "grasp reflex" are capable of analysis into two distinct and separate components, the one volitional and the other reflex. They concluded that the grasping movements in response to objects seen, or to contacts felt in the palm of the hand (sometimes associated with movements of the hand and arm through space) are voluntary responses to those visual and tactile stimuli, to which grasping is a natural and satisfactory response. They are, in fact, volitional movements occurring in individuals, whose psychomotor functions have been reduced by disease of the brain to the "least voluntary and most automatic" movements of Hughlings Jackson. They confined the reflex component to the involuntary tightening and persistence of the grasp when the object is pulled away, which occurs when and only when the muscles are subjected to the proprioceptive stimulus of stretch. This is quite outside the patient's control, and tactile or visual stimuli are not sufficient to produce it.

An unusual case is presented here of the grasp reflex associated with an arm so severely hemiplegic that voluntary grasping and clenching of the hand was impossible, thus enabling several conclusive observations to be made on the significance of tactile and proprioceptive stimuli when the volitional factor has been excluded.

\section{Personal Case}

T. G., male, aged 64, was admitted to the National Hospital under the care of Dr. Carmichael on 27th May, 1937. 
In December, 1923, in the Victoria Infirmary, Glasgow, a left frontal meningioma had been removed. He was left with a slight residual hemiplegia on the right side, which persisted almost unaltered till 4 months before admission. He could move his fingers fairly freely and he could always open and close his hand without difficulty or delay when it was empty. However, this right hand, some 6 months after the operation, developed a habit of clutching hold of objects of its own accord, such as the lapel of his coat, the edge of his trouser pocket, or the bed-clothes. This distressed him so much that in order to prevent it, he used to wear a glove most of the time. At other times he would keep his hand in his pocket, holding a coin or a key. "I never felt comfortable unless I had something in my hand. I felt easier holding a coin in my hand." He also experienced great difficulty letting go of things with this hand, and he frequently had to use his other hand to help. For instance, he was able to hold a shovel firmly and shovel up heavy things, but in order to leave go, he had to use his left hand " to undo" his right hand. "I used to carry a bucket of coal in each hand. I could let go of the bucket in my left hand easily, but not the one in my right. I had to pull my fingers away with the other hand."

Four months previous to admission he had a right-sided Jacksonian epileptic attack associated with loss of consciousness, and since then his right arm has been so weak that he could not clench his hand voluntarily. The hand no longer groped after or grasped things near it. However, the hand of its own accord still continued to close on objects placed in the palm and he continued to have great difficulty in releasing the grasp.

Examination revealed a marked right hemiplegia affecting the arm more than the leg. The fingers could only be flexed slowly and feebly to the extent of bringing the tips lightly into contact with the palm, but no pressure could be exerted. The movement was slow and tremulous. The patient was quite unable to imitate voluntarily the strong automatic tightening of the grasp that was immediately initiated by a slight stroking movement. No voluntary extension of the fingers or thumb was possible. All forms of sensation, including position, passive movement, two point discrimination, and stereognosis were unimpaired. There was no apraxia. There was no forced grasping or groping in relation to objects coming near or into contact with parts of the hand other than the palm.

\section{The Grasp Reflex}

A light stroke with a piece of cotton-wool produced a maximum response when applied to the skin over the palmar aspects of the metaphalangeal joints and the proximal part of the first interspace, between the finger and thumb. A response was less easily obtained over the remainder of the palmar aspect of the hand, fingers, and thumb as far as the distal interphalangeal joints. The involuntary grasp was most easily provoked when an object was introduced between the thumb and first finger with the hand half clenched. The further the fingers were passively extended the more difficult was it to produce a response. When the fingers were held fully extended by the observer no stimulation of the palm, even firm pressure, produced any response. The same applied to stimulation in the first interspace when the thumb was held passively in the fully abducted position. If the fingers were held fully extended and the thumb left free, stroking the skin in the first interspace with cotton-wool produced a feeble tremulous adduction of the thumb.

A finger, a pencil, or a large wad of cotton-wool introduced into the palm of the hand-especially in the radial half-caused the hand to form a light 
comfortable grasp round it. This movement had all the attributes of a true grasp, and was more than mere flexion of the fingers. There was no turning movement of the forearm or arm towards the object. So long as the object lay motionless in the hand the grasp was only loose and the observer could easily insert his fingers beneath the tips of the patient's fingers and passively extend them. A slight movement of the object-especially a withdrawal movement radially-even in the case of the purely cutaneous stimulus of cottonwool, produced an immediate increase in the contraction of the flexors and the object was grasped more tightly. If a slight continuous tension was held on the object in the direction of withdrawal, a coarsely irregular tremulous grasp was kept up until the tension was released. As the strength of the pull was increased, so the grasp became firmer, until finally, in the case of the observer's finger, the patient was nearly pulled out of bed. The grasp was relaxed at once, without any delay, the moment tension ceased. There were no after-movements of the hand.

Stimulation of the sides of the finger and thumb in the first interspace produced adduction of the thumb alone. Introduction of an object into the ulnar half of the palm caused a feeble grasping movement, confined to the two ulnar fingers.

If the observer's finger or a pencil was pulled away in a distal direction so as to throw a stretch on the flexor tendons, then the flexors went into spasm and the hand "clung" to the object. The strength of the "cling" increased with the tension of the pull and became so strong that the patient could be pulled into the sitting position in this way. This " clinging" could be obtained by pulling on all the fingers together or on any one finger separately.

The following observations were made after a " novocaine block" of the median, ulnar, and radial nerves at the wrist. 1: There was complete loss of appreciation of pinprick, cotton-wool strokes, and light touches with a match-stick over the dorsal and palmar aspects of the whole hand and all the fingers. 2: Firm pressure with the head of a match on the muscles of the palm and palmar aspect of the fingers was appreciated. 3: The finest and most delicate changes in position were appreciated in the joints of all fingers. 4 : No grasp was provoked by introducing into the palm of the hand from the radial side, cotton-wool, a match-stick, the rounded head of a hat-pin, or a pencil. Gentle rubbing of the skin of the palm with any of these articles and withdrawal produced no grasping - a stimulus which invariably produced a response in the hand before and again two hours after the anæsthetization. 5: The observer's finger could be introduced carefully into the palm of the semi-clenched hand, withdrawn, rubbed gently backwards and forwards, rotated, and even gently circumducted so as to produce visible displacement of the phalanxes without any grasping movements occurring. 6: If the observer's finger or a pencil was inserted into the semi-closed hand from the radial side and withdrawn in such a way as to press firmly on the adjacent muscles and tendons, a feeble grasping movement was obtained. 7 : A strong grasp could be obtained by introducing two fingers between the finger and thumb and withdrawing it with a slight twist in a distal direction in such a way as to 
put a considerable stretch on the flexors of the thumb and forefinger. $8:$ A strong " cling " could still be obtained, as before anæsthetization, by " pulling " on the semi-flexed fingers.

No change in the nature or strength of the grasp was produced by changes of posture of the head relative to the trunk or of the posture of the body in space. This observation suggests that the grasp reflex is not part of the neck or labyrinthine righting reflexes of Magnus and de Kleyjn.

\section{Discussion}

For 14 years after the removal of a left frontal meningioma, this patient had a mild right hemiplegia, associated with well-marked grasping movements and difficulty in relaxing the grasp, illustrating very prettily most of the phenomena of the grasp reflex described by previous observers.

Four months ago, following a fit, there was a great increase in the loss of power in the arm, probably associated with destruction of further brain tissue by vascular changes. The weakness became so severe that he could only close his hand feebly with a slow, tremulous movement to the extent of bringing the tips of the fingers lightly into contact with the palm. He was quite unable to clench his hand or voluntarily imitate the grasp which could be so readily obtained reflexly. His hand no longer groped and grasped after things near it or coming into contact with parts other than the palm, and all turning movements of the wrist, forearm, or arm towards the object disappeared. The loss of these elements is compatible with Walshe's hypothesis that these are volitional and belong to the "least voluntary and most automatic movements," though it does not follow that this is necessarily the true explanation.

Although, as a rule, when such severe hemiplegia is present the grasp reflex disappears, in this case the introduction of an object into the palm of the hand provoked an involuntary closure, the characteristics of which are fully described in the case report. This grasp was unquestionably quite outside the volitional control of the patient, who could not prevent it and who was quite unable to imitate it voluntarily.

A light stroke with cotton-wool invariably produced this reflex grasp, but after a regional nerve block with novocaine this stimulus was no longer effective. While it was thus demonstrated that a tactile stimulus alone was sufficient to produce a response, a reflex grasp could still be obtained after the anæsthetization by introducing the observer's finger in such a way as to press on the surrounding muscles and tendons, especially if a slight twist was made so as to throw a stretch on the flexor tendons. It was therefore conclusively shown, in this case, that an involuntary reflex grasp could be produced in response to either tactile or proprioceptive stimuli alone, and it would appear that in the ordinary way with the unanæsthetized hand, the reflex grasp is a response to a combination of both tactile and proprioceptive impulses set up by the object entering the hand.

It was also found that any attempt to withdraw or pull away a piece of cottonwool from the palm immediately produced a tightening of the grasp, which the patient could not prevent, a response which could no longer be obtained after 
anæsthetization. The withdrawal of a firmer object, such as the observer's finger, in such a way as to produce pressure and stretch on the surrounding muscles and tendons produced a still more powerful tightening of the grasp, which persisted after anæsthetization. It appears in this case, therefore, that in the same way as in the initiation of the grasp reflex, so the tightening and persistence of the grasp on attempting to withdraw an object is reflexly produced and maintained by both tactile and proprioceptive afferent stimuli.

I wish to express my indebtedness to Dr. E. A. Carmichael for his permission to publish this case and for his kind help and advice.

\section{REFERENCES}

Adie, W. J., and Critchley, M. (1927). Brain, 50, 142.

Walshe, F. M. R., and Robertson, E. G. (1933). Ibid., 56, 40. 